J. GROLL, Leiden.

CORN'. DE GROOT, 's Gravenhage.

J. H. DE GROOT, 's Gravenhage.

J. J. M. DE GROOT, 's Gravenhage.

Jhr. c. de giJselaar, 's Gravenhage.

Mr. H. F. L. hamelbeizg, Arnhem.

Mr. G. G. VAN HARENCARSPEL, 's Hage.

L. K. HARMSEN, Leiden.

D. hartevelt, Leiden.

Jhr. C. hartsen, Amsterdam.

J. J. hassemman, Tiel.

w. van hasselt, Amsterdam,

Mr. J. HeEMSKerk Az., 's Gravenhage.

Mr. A. heEMSKerk az., Caïro.

B. HELDRING, Amsterdam.

Mr. J. E. HENNY, Amsterdam.

J. K. VAN DER HEIJDEN, 's Grivenharge!

F. F. HEINEE, "s Gruvenhage.

i. MIRBIXK, Zutfen.

$\mathrm{N}$, Horstgde, 's Gravenhage.

Prof. Dr. J. J. DE holitander, Breda. 6. DU IHU VAN BR̃BsT HOLLE, 's Hage,

Dr. ‥ тH. Hovт8Ma, Leiden,

3. HบDG $\mathrm{I} \% \mathrm{z}$, Amsterinm.

H. Q. HuMus, 's Gravenhage.

Jir. Mr, j, HUYDECORER VAN MAAR8BEYEFA, Maaraseveen.

i. A. TNsixom, Bantr.

Mr. E, H. 'S JACOB, Utreolit.

F. W. JANBEN, Amsterdam,

c. A, J, L. JEEKFi, 's Gravenbige.

Dr, $\overline{7}$, A. JENIINK, Leiden.

c. J. M. JONGKIXIT CONTRCK, $\bar{W}_{\text {ageningen. }}$

A. 0, Jooster, Amsierdam,

Frof, Dr, A. W, T. jurxbou, Delft,

Prof. Dr, c. M, KAy, Amsterdam,

Prot. Dr, ㅍ. KEN

c. E. TAN KFsTEREx, 's Grivenhuge.

E. 18. KIMLSTRA, 's Gravenhuge.

W, F. YaA ERT TAALMaN EIF, 'sHage.

II. C. BLIAK GRT, Leiden.

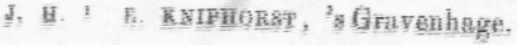

J. C. DE KOCK VAN LEEUWEN, 's Hage.

C. P. J. VAN KOETSVELD, Rotterdam (adres: L. van Koetsveld.)

Prof. Dr. E. F. KRUIJYF, Groningen.

J. A. Krolut, Pinang.

J. KUYPER HzN., 's Gravenhage.

N. D. LAMmers van toorenbURG, 's Gravenhage.

Mr. J. w. van lansberge, Brummen.

Mr. H. o. VAN DER LINDEN, Dordrecht.

E. H. LASONDER, Utrecht.

c. J. LEENDERTZ, Leiden.

Mr. H. D. Levyssohn norman, 's Hage.

o. J. H. Graaf van LIMbURG stirum, 's Gravenhage.

Prof. Mr. P. A. vAN DER LITH, Leiden.

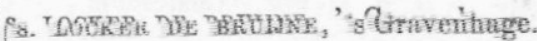
Mr. H. HOPE LOUDON, 's Gravenhage. Prof. Mr. J. DE Lourwe, Utrecht. D. MaAnschaLK, 's Gravenhage.

Mr. J. c. DE MrAREz OTENs, 's Gravenhage. Prof. Dr. k. мartin, Leiden.

Dr. B. $z$. Matrites, 's Gravenhage.

Mr. P. Alrme arees, 's Gravenhage.

Prof. Ј. Ј. Meinsma, Delft.

Avgugr mereitz, Amsterdam.

Dr. k. w. I. MoNmus, Schiedam.

H. MULLEE sZx., Rotterdam.

A. D. VAX DER GON NETBCHER, 's Gravenhage.

F. M. NETschæm, 's Gravenhage.

Prof. G. E. NLemaxx, Delft.

Jhr. Mr. 0. E. vax nIsPex, 's Hage.

M. NIנHOFE, "s Gravenhage.

Mr. 0. W, stall xumax, 's Gravenhage.

A. P. M. YAA OOHDT, Leiden.

H. j, 008Tixg, Assen.

c. $x$. PABST, 'sGravenhage.

Mr. I, T. PAHUD ${ }^{*} \mathrm{DE}$ MoRTAyges, Arnhem,

W, PAHUD DE Montaxges, 's Hage.

Mr. I. 6, FATIJS, '8 Gravenhage. 
Mr. D. L. F. DE PAULY, Florapark, Haarlem.

M. P. PELs, Amsterdam.

M. T. H. PERELAER, 's Gravenhage.

Prof. Mr. n. G. PIERson, Amsterdam. Prof. Dr. J. PIjNappel gzN., Leiden.

J. G. Plate JR., Leiden.

w. PLEYTE, Leiden.

Dr. FRANçoIs P. L. POLLEN, Scheveningen. A. POMPE, Breda.

Jhr. $\boldsymbol{F}$. POMPE VAN MEERDERVOORT,

's Grarenhage.

v. w. Posthumus, Amsterdam.

н. PRANGe, Utrecht.

A. PRUYS vaN DER hOEven, 's Hage.

A. S. PRANSEN VAN DE PUTTE; Arnhem.

Jhr. Mr. J. K. W. QUARLES VAN UFYORD,

's Gravenhage.

Jhr. Mr. w. van RAPPARD, 's Hage.

M. A. VAN RHEDE VAN DER KLOOT,

's Gravenhage.

Jhr. Mr. G. C. J. van Reenen, 's Hage. J. G. P. RIEDEL, Utrecht.

Dr. w. N. DU RIEU, Leiden.

Jhr. Mr. J. ROËIL, 's Gravenhage.

J. w. Roskes, Rotterdam.

C. RUEB CZ., Rotterdam.

Dr. E. VAN RIJCKEVorsel, Rotterdam. A. W. J. FARNCOMBE SANDERs, 's Hage. W. Schagen van LeeUwen, Delft.

Mr. J. c. J. vAN DER SCHALK, NoordwijkBinnen.

D. SCHeltema, Haarlem.

Prof. Dr. G. Schlegel, Leiden.

Prof. H. SCHLEgel, Leiden.

J. $\boldsymbol{F}$. L. SCHNEIDER, Delft.

Dr. J. SEMarelnN, 's Gravenhage.

a. M. Servatius, Terwolde bij Deventer. Mr. c. J. sICKEsz, Laren.

Jhr. J. D. sIx, 's Gravenhage.

Mr. L. A. J. w. Baron SLOET VAN DE Beele, Arnhem.
Dr. H. sMedrsg, Haarlem.

н. зон. SMID, Dennenoord, Laren.

JOH. F. SNElleman, Amsterdam.

Dr. c. snovck hurgronje, Leiden.

Prof. J. SPanjaAkd, Delft.

Dr J. s. SPEIJER, Amsterdam.

J. P. SPRENGER VAN EIJK, 's Gravenhage.

Dr. N. P. VAN DER sTOK, Rijswijk

(Buitengedachten).

p. DE stoppelaAR, Leiden.

Jhr. Mr. v. DE stuers, 's Gravenhage.

Jhr. c. A. van sypesteisn, 's Gravenbage.

A. W. SIJTHоF, Leiden.

Mr. J. P. R. TAK VAN POORTVLIET,

's Hage.

Jhr. W. T. TEDING VAN BERKHOUT,

's Gravenhage.

P. E. TEgelberg, Amsterdam.

Mr. G. vaN THENHoven, Amsterdam.

N. Trakranen, Amsterdam.

Prof. Dr. J. J. P. valeton, Groningen.

Mr. s. a. vening MeInesz, Rotterdam.

D. D. vETH, Leiden.

T. P. VIRULY, Leiden.

c. A. M. VAN VLIET, 's Gravenhage.

Dr. J. VAN DER VLIET, Haarlem.

B. R. F. VAN vLIJMen, Nijmegen.

JoOST vaN volLENHOven, Rotterdam.

Mr. J. A. G. Baron DE vos vaN STEENWIJK, Utrecht.

Prof. A. c. vReede, Leiden.

E. DE WAAL, 's Gravenhage.

w. A. VAN WALCHEREN, 's Gravenhage.

M. J. WALler, Amsterdam.

Dr. s. J. Warren, Dordrecht.

A. в. WASZEKLEWICZ, 's Gravenhage.

A. W. P. WEITZEL, 's Gravenhage.

G. F. Westerman, Amsterdam.

WESTEROUEN VAN MEETEREN, 'Amsterdam c. F. W. WICHERS VAN KERCHEM, Leiden.

Prof. Dr. c. E. A. Wichmann, Utrecht. 
J. А. в. Wiselius, Parijs, rue de l'Université, 107.) WILLEM WIJT, Rotterdam. Dr. G. A. WLKEN, Leiden.

J. WOLBERS, Utrecht. D. G. E. WOLTERBEeK MULLER, 's Hage.
J. wüste, Amsterdam.

Jhr. Mr. H. C. vaN DER WIJCK, 's Hage. Jhr. Mr. H. VAN DER WIJCK, 's Hage. Dr. тн. сн. L. wijnmalen, 's Hage. s. B. zeVERIJN, Amsterdam.

\section{CORRESPONDEERENDE LEDEN.}

C. H. B. von ROSENBerg, 's Gravenhage.

P. A. TIELE, Utrecht.

J. C. NEURDENBURg, Rotterdam.

C. A. A. de Magnin, Parijs (Avenue Madrid, no. 11 Neuilly S. Seine.)

\section{NEDERLANDSCH OOST-INDIË.}

\section{GEWONE LEDEN.}

G. C. D'ABo, Landhuurder, Batoe Djamoes (Soerakarta).

J. W. H. ADÈr, Predikant te Batavia.

J. A. aeckerlin, Secretaris van de residentie Benkoelen (Sumatra).

J. e. Albrecht, President van de Weeskamer, Bibliothecaris v. h. Bataviaasch Genootschap van Kunsten en Wetenschappen, Batavia.

Jhr. F. H. P. vAN ALPHEN, Kommandant van de Zeemacht in N.-I., Batavia.

H. M. ANDRÉE WILtens, Vice-president van den Raad van Ned.-Indië, Batavia.

C. BAUMGARTEN.

Mr. N. P. VAN DEN BERg, President van de Javasche Bank, Batavia

Mr. L. W. c. van den berg, Ambtenaar voor de Inlaudsche talen, Batavia.

J. BoDdÉ, Zendeling te Tengah-Sonder (Menado).

є. возсH, Assistent-resident voor de policie, Padang (Sumatra's Westkust).

J. A. H. BREYManN, contrôleur te Koemering oeloe (Moeara doea).

w. p. van charante, President van het Liefdadigheids-gesticht enz. te Depok (Buitenzorg).

Mr. J. A. VAN DER CHIJs, Ambtenaar belast met het toezicht over 't archief, Batavia.

Mr. J. W. $y$ vohen stuart, Advocaat, Semarang.

A. J. W. vaN DELDEN, Directeur van de Spaarbank, Batavia.

J. A. DEZENTJE, Landhuurder, Ampal, residentie Soerakarta. 
s. vaN DISsEL, Inspecteur van het inlandsch onderwijs, Batavia.

P. A. L. E. VAN DIJK, Contrôleur le klasse te Si-Lindoeng (Toba-Bataklanden).

P. VAN DIJK, Hoofdmijningenieur, Batavia.

J. ENNEN, 2e onderwijzer aan de kweekschool, Fort de Kock.

Mr. J. W. Essers, Raadsheer in het Hooggerechtshof, Batavia.

r. rockens JR., Contrôleur 2e klasse, Poerwakarta.

D. Gerth van wiJk, Leeraar in de Maleische taal, Commissaris van het Instituut, Batavia.

Dr. J. H. F. SOLLEWYN GELPKE, Hoofd-inspecteur der kultures, Buitenzorg.

G. P. H. H. GONGGRIJP, Algemeen ontvanger, Batavia.

w. P. Groeneveldt, Secretaris van het Dep. onderwijs enz., Batavia.

s. GRaAfland, Directeur der Kweekschool, Tanawangko (Menado).

A. J. A. Grasyland, Ambtenaar te Kebajoeran in de afd. Meester-Cornelis, Batavia.

Dr. J. G. H. GUNNING, Ambtenaar voor de inlandsche talen, Soerakarta.

Dr. c. Gutteling, Geneesheer, Batavia.

Mr. J. а. валкмах, Redacteur Bat. Handelsblad, Batavia.

J. наввема, Hoofdonderwijzer, Amboina.

A. HAGA, Kolonel, chef Generale staf, te Batavia.

G. J. harrebomée, Contrôleur le klasse te Kotan Nopan (afd. Rau, Sumatra's Westkust).

A. L. Van hasselt, Secretaris van den Raad van Indië, Commissaris van het Instituut, Batavia.

н. van hedckeldm, Assist.-resident te Padang Sidempoean (Sumatra's Westkust).

J. HEIJTING, Inspecteur van financiën, Batavia.

Dr. P. Heidting, Predikant te Poerworedjo.

в. HoEtrnk, Tolk voor de Chineesche taal te Deli Médan (Sumatra's Oostkust).

G. W. w. C. Baron van hoevelu, Contrôleur le klasse te Kajoetanam, afd.

Priaman (Sumatra's Westkust).

w. HOEzoo, Zendeling, Semarang.

Dr. J. w. HofrmanN, Geneesheer, Buitenzorg.

K. ғ. HOLLE, Adviseur, Waspada (Preanger Regentschappen).

Jhr. H. W. F. HORA SICCAMA, Resident, Batavia.

Dr. D. w. HоRst, Contrôleur le klasse, te Kroë, resid. Benkoelen.

P. A. JANsz, Zendeling-leeraar, Depok.

E. W. DE JONG, Ambtenaar voor de burgerlijke dienst in N.-I. te Tjitjoeroeg, (Preanger Regentschappen).

Mr. т. H. DER KINDEREN, Lid van den Raad van Ned.-Indië, Batavia.

J. KNebel, Contrôleur le klasse, Pasoeroean.

Jhr. ш. н. ш. ре коск, Secretaris van de Padangsche Bovenlanden, Fort de Kock.

P. J. Kooreman, Secretaris der residentie Oostkust van Sumatra, Bengkalis.

G. J. VAN KOOTEN, le Luitenant artillerie N. I., Batavir. 